\title{
On Connectivity of Fatou Components concerning a Family of Rational Maps
}

\author{
Junyang Gao ${ }^{1}$ and Gang Liu $^{2}$ \\ ${ }^{1}$ School of Science, China University of Mining and Technology, Beijing 100083, China \\ ${ }^{2}$ Department of Mathematics and Computational Science, Hengyang Normal University, Hengyang 421002, China \\ Correspondence should be addressed to Gang Liu; liugangufo@tom.com
}

Received 17 November 2013; Revised 12 February 2014; Accepted 12 February 2014; Published 23 March 2014

Academic Editor: Irena Rachůnková

Copyright (c) 2014 J. Gao and G. Liu. This is an open access article distributed under the Creative Commons Attribution License, which permits unrestricted use, distribution, and reproduction in any medium, provided the original work is properly cited.

\begin{abstract}
I. N. Baker established the existence of Fatou component with any given finite connectivity by the method of quasi-conformal surgery. M. Shishikura suggested giving an explicit rational map which has a Fatou component with finite connectivity greater than 2. In this paper, considering a family of rational maps $R(z, t)$ that $\mathrm{A}$. F. Beardon proposed, we prove that $R(z, t)$ has Fatou components with connectivities 3 and 5 for any $t \in(0,1 / 12]$. Furthermore, there exists $t \in(0,1 / 12]$ such that $R(z, t)$ has Fatou components with connectivity nine.
\end{abstract}

\section{Introduction and Main Results}

By Sullivan's theorem [1], each Fatou component of a rational map is eventually periodic. Moreover, for periodic Fatou components, there are only four possibilities: attracting basin, parabolic basin, Siegel disk, and Herman ring. Attracting basins and parabolic basins are either simply connected or infinitely connected, a Siegel disk is simply connected, and a Herman ring is doubly connected. However, for nonperiodic Fatou components, the corresponding connectivity may be bigger than two.

For any given $n \in \mathbb{Z}^{+}$, Baker et al. [2] proved that there exists a rational map $R$ which has a Fatou component with connectivity $n$ by the method of quasiconformal surgery. M. Shishikura suggested giving an explicit example such that it has a Fatou component with finite connectivity greater than two. Beardon [3] investigated the family of rational maps as follows:

$$
R(z, t)=\frac{z^{2}\left(1+t^{12} z^{3}\right)}{(1-t z)^{3}\left(1-t^{4} z\right)}, \quad(z \in \overline{\mathbb{C}}, t \in \mathbb{R}) .
$$

He proved the following result.
Theorem A. For sufficiently small $t>0$, there exists a Fatou component $D$ of $R(z, t)$ with connectivity three or four.

At the same time, he claimed that one may be able to compute the connectivity of $D$ by further discussion. Qiao and Gao [4] verified that $D$ has connectivity three for $t \epsilon$ $\left(0,10^{-4}\right)$. Moreover, for any given positive integer $n$, two different families of rational maps were constructed such that one of them has a Fatou component with connectivity $n$ (see $[4,5])$. However, the degree of rational maps satisfies with those conditions are increased as the number $n$ increases. As the first step to study the problem of connectivity number of Fatou components in rational maps space with fixed degree, we just investigate the connectivity of any other Fatou component of $R(z, t)$ as the real parameter $t$ varies. In fact, we have the following results.

Theorem 1. Suppose that $R(z, t)$ is defined as in (1); then we have the following.

(1) For any $t \in(0,1 / 12]$, there exist two Fatou components $D_{t}$ and $\widetilde{D_{t}}$ of $R(z, t)$ with connectivities three and five, respectively. Moreover, $R: \widetilde{D_{t}} \rightarrow D_{t}$ is an unbranched covering. 
(2) There exists $t \in(0,1 / 12]$ such that $R(z, t)$ has one Fatou component with connectivity nine.

Remark 2. In order to draw the graphs of Julia sets and Fatou components of such rational maps in complex plane, we consider its conformal conjugate. Let $g(z)=1 / z$, and put $\widetilde{R}(z, t)=g \circ R \circ g^{-1}(z, t)=\left(z(z-t)^{3}\left(z-t^{4}\right)\right) /\left(z^{3}+t^{12}\right)$. It is easy to see that $\infty$ is a superattracting fixed point of $\widetilde{R}(z, t)$ for any $t \in(0,1 / 12]$. By Figure $1, R(z, t)$ has Fatou components with connectivities three, five, and nine for some $t \in(0,1 / 12]$ since $R(z, t)$ and $\widetilde{R}(z, t)$ have the same dynamical properties. Furthermore, by Figure 2 , we know that $R(z, 0.004355)$ has Fatou components with connectivities eight and fourteen, and we conjecture that for any large integral $N \in \mathbb{N}$, there exists $t_{0} \in(0,1 / 12)$ such that $R\left(z, t_{0}\right)$ has a Fatou component with its connectivity bigger than $N$.

\section{Preliminary Lemmas}

For the fundamental concepts and classical results of iteration theory of rational maps, see $[2,3,6,7]$. In order to prove Theorem 1, we need the following four lemmas. Except for Lemma 3 , the others are certain modifications of results which have been verified in [3].

Lemma 3 (see [8, Proposition 2.5]). Let $f$ be a rational map of a degree larger than one, and let $\left\{U_{i}\right\}_{i=1}^{p}$ be its one (super)attractive or parabolic cycle of periodic Fatou components. If one of $U_{i}(i=1, \ldots, p)$ is not simply connected, then $\cup_{i=1}^{p} U_{i}$ contains at least two different critical values for critical points in itself.

In what follows, $t \in(0,1 / 12]$ in $R(z, t)$. It is easy to see that $z=0$ (resp., $z=\infty$ ) is a superattracting (resp., repelling) fixed point of $R(z, t)$. Let $D_{00}$ be the Fatou component that contains $z=0$.

Lemma 4. The nonzero critical points of $R(z, t)$ lie outside the circle $\{z:|z|=3\}$.

Proof. $R(z, t)$ has exactly eight critical points, that is, $0,1 / t, 1 / t$ and $\xi_{j}(j=1,2,3,4,5)$, which are the solutions of the equation $R^{\prime}(z, t)=0$. Obviously, $z=1 / t$ lies outside the circle $\{|z|=3\}$. By a calculation, each $\xi_{j}(j=1,2,3,4,5)$ satisfies the following equation:

$$
2+t z \quad H(z, t)=0,
$$

where $H(z, t)=t^{16} z^{4}-2 t^{12}\left(1+2 t^{3}\right) z^{3}+5 t^{11} z^{2}-2 t^{4} z+1-t^{3}$. Putting

$$
M=\sup \left\{|H(z, t)|:|z| \leq 3, t \in\left(0, \frac{1}{12}\right]\right\},
$$

we can deduce that

$$
M \leq 81 t^{16}+54 t^{12}\left(1+2 t^{3}\right)+45 t^{11}+6 t^{4}+t^{3}+1 \leq 2 .
$$

By (2), the nonzero critical points of $R(z, t)$ lie outside the circle $\{z:|z|=3\}$.
Lemma 5. $D_{00}$ is simply connected and $\{z:|z| \leq 1 / 2\} \subset$ $D_{00} \subset\{z:|z| \leq 3 / 2\}$.

Proof. For $z \in K=\{z: 0<|z| \leq 3 / 2\}$, we have

$$
\begin{aligned}
\left|\frac{R(z, t)}{z^{2}}\right| & \leq \frac{1+t^{12}|z|^{3}}{(1-t|z|)^{3}\left(1-t^{4}|z|\right)} \\
& \leq \frac{1+(3 / 2)^{3} t^{12}}{(1-(3 / 2) t)^{3}\left(1-(3 / 2) t^{4}\right)}<\frac{3}{2} \\
\left|\frac{R(z, t)}{z^{2}}\right| & \geq \frac{1-t^{12}|z|^{3}}{(1+t|z|)^{3}\left(1+t^{4}|z|\right)} \\
& \geq \frac{1-(3 / 2)^{3} t^{12}}{(1+(3 / 2) t)^{3}\left(1+(3 / 2) t^{4}\right)}>\frac{2}{3} .
\end{aligned}
$$

If $|z| \leq 1 / 2$, then $|R(z)| \leq(3 / 2)|z|^{2} \leq(3 / 4)|z|$; we can deduce that $\{z:|z| \leq 1 / 2\} \subset D_{00}$.

Suppose that $D_{00}$ meets the circle $\{z:|z|=3 / 2\}$; take a point $\omega \in D_{00} \cap\{z:|z|=3 / 2\}$ and join $\omega$ to the origin by a curve $\sigma \subset D_{00}$. It is easy to see that $R^{n} \rightarrow 0$ uniformly on $\sigma$; then there exists a unique positive integer $k$, such that $R^{k}(\sigma)$ meets the circle $\{z:|z|=3 / 2\}$, but $R^{n}(\sigma)$ does not meet $\{z:|z|=3 / 2\}$ for $n>k$. Let $\zeta$ be a point where $R^{k}(\sigma)$ meets $\{z:|z|=3 / 2\}$; we have

$$
|\zeta|>|R(\zeta)|>\frac{2}{3}|\zeta|^{2}=|\zeta|
$$

It is a contradiction and thus $D_{00} \subset\{z:|z| \leq 3 / 2\}$. Obviously, $D_{00}$ contains only one critical point $z=0$ by Lemma 4 ; then $D_{00}$ is simply connected by Lemma 3 .

Lemma 6. $R^{-1}\left(D_{00}\right)$ only consists of two Fatou components, that is, $D_{00}$ and $D_{01}$, which contains a triply connected domain $\Omega$. Here, $\Omega=\left\{z: 1 / 2 t^{4}-1 / t \leq|z| \leq 3 / 2 t^{4}+1 / t,\left|z-1 / t^{4}\right| \geq\right.$ $\left.1 / 2 t^{4}\right\}$.

Proof. Take $z \in \Omega$; by a simple calculation, we have

$$
\begin{aligned}
t|z|-1 & \geq \frac{1}{2 t^{3}}-2>800, \\
\frac{1}{2}-t^{3} & <t^{4}|z|<\frac{3}{2}+t^{3}, \\
\left|1-t^{4} z\right| & \geq \frac{1}{2}
\end{aligned}
$$

It is easy to see that $1 /(t|z|-1)<1.002 / t|z|$; we have

$$
\begin{aligned}
\left|\frac{z^{2}\left(1+t^{12} z^{3}\right)}{(1-t z)^{3}}\right| & \leq \frac{|z|^{2}\left(1+t^{12}|z|^{3}\right)}{(t|z|-1)^{3}} \\
& <(1.002)^{3} \frac{|z|^{2}\left(1+t^{12}|z|^{3}\right)}{(t|z|)^{3}}<3 t .
\end{aligned}
$$

Moreover, $R(\Omega) \subset\{z:|z| \leq 6 t\} \subset\{z:|z| \leq 1 / 2\}$. By Lemma 5, we have $R(\Omega) \subset D_{00}$ and $\Omega \subsetneq D_{00}$, and there exists 


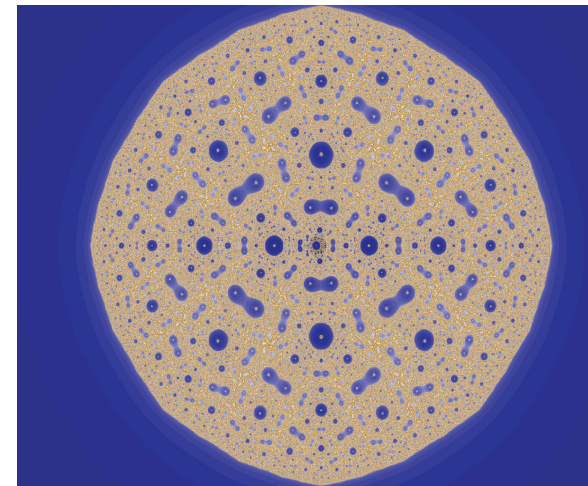

(a)

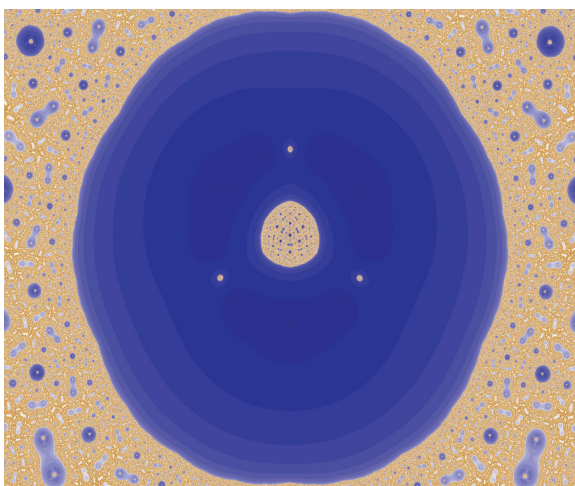

(c)

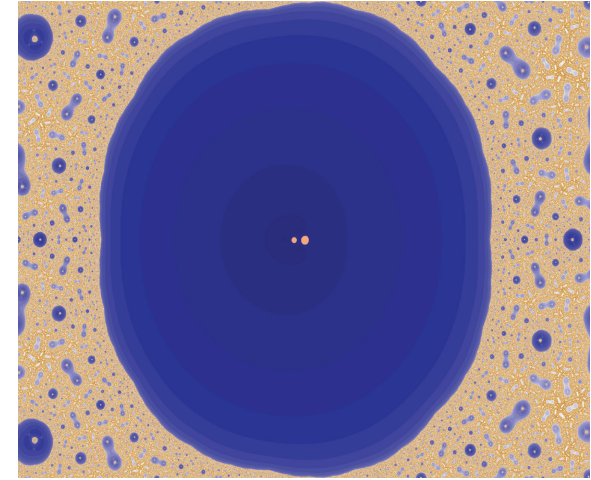

(b)

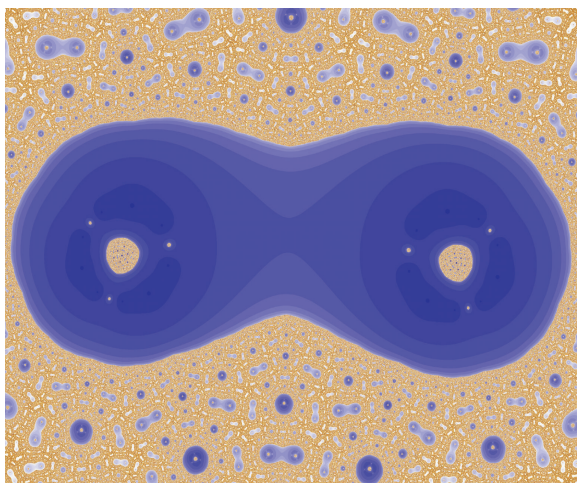

(d)

Figure 1: The Julia set $J(\widetilde{R}(z, 1 / 20))$ and three Fatou components of $\widetilde{R}(z, 1 / 20)$ with connectivities 3,5 , and 9, respectively.

at least one Fatou component $D_{01}\left(\neq D_{00}\right)$ of $R^{-1}\left(D_{00}\right)$. Noting that there are three (resp., two) zeros of $R(z, t)$ in $\Omega$ (resp., $\left.D_{00}\right)$, this implies that $R^{-1}\left(D_{00}\right)=D_{00} \cup D_{01}$ and $D_{01} \supset \Omega$.

\section{Proofs}

Let $D \subset \mathbb{C}$ be a bounded Fatou component of $R(z, t)$. We denote the connectivity of $D$ by $n(D)$ and the unbounded component of $\overline{\mathbb{C}} \backslash D$ by $\operatorname{Out}(D)$. Let $\operatorname{Int}(D)=\overline{\mathbb{C}} \backslash \operatorname{Out}(D)$ and $\left.d\right|_{D}:=\operatorname{deg}(R: D \rightarrow R(D))$. Moreover, we say that a component of $R^{-n}(D)(n \in \mathbb{N})$ is a component $\widetilde{D}$ such that $R^{n}(\bar{D})=D$. We say that $D$ surrounds a point $z(\in \mathbb{C}$ ) (or a domain $U(\subset \mathbb{C}))$ if it satisfies $z \in \operatorname{Int}(D)(\operatorname{or} U \subset \operatorname{Int}(D))$ and denote by $D \cup z$ (or $D \cup U$ ). Denote the number of zeros and poles of $R(z, t)$ in the interior of Jordan curve $\gamma \subset \mathbb{C}$ by $N(R, \gamma)$ and $P(R, \gamma)$. In order to prove Theorem 1 , we need the following propositions. Considering the connectivity of $D_{01}$ in Lemma 6, we have the following result.

Proposition 7. $D_{01}$ is a triply-connected domain.

Proof. By a simple calculation, we have

$$
R^{\prime}(z, t)=\frac{z Q(z, t)}{(1-t z)^{4}\left(1-t^{4} z\right)^{2}} .
$$

Here

$$
\begin{aligned}
Q(z, t)= & t^{17} z^{5}-2 t^{13}\left(1+2 t^{3}\right) z^{4}+5 t^{12} z^{3} \\
& -2 t^{5} z^{2}+t\left(1-t^{3}\right) z+2 .
\end{aligned}
$$

Note that

$$
\begin{aligned}
Q\left(-\frac{2}{t}+5 t^{2}, t\right)= & -t^{3}+35 t^{6}-122 t^{9}+524 t^{12}-910 t^{15} \\
& -1775 t^{18}+7750 t^{21}-8750 t^{24} \\
& +3125 t^{27}<0, \\
Q\left(-\frac{2}{t}+6 t^{2}, t\right)= & 42 t^{6}-144 t^{9}+648 t^{12}-1560 t^{15} \\
& -1800 t^{18}+12960 t^{21}-18144 t^{24} \\
& +7776 t^{27}>0,
\end{aligned}
$$

we can deduce that there exists a point $c_{0} \in\left(-2 / t+5 t^{2},-2 / t+\right.$ $\left.6 t^{2}\right)$ such that $Q\left(c_{0}, t\right)=0\left(c_{0}\right.$ is a critical point of $\left.R(z, t)\right)$. Note that $\left(-2 / t+5 t^{2},-2 / t+6 t^{2}\right) \subset(-2 / t,-1 / t)$ and

$$
R\left(-\frac{x}{t}, t\right)=\frac{x^{2}\left(1-t^{9} x^{9}\right)}{t^{2}(1+x)^{3}\left(1+t^{3} x\right)},
$$




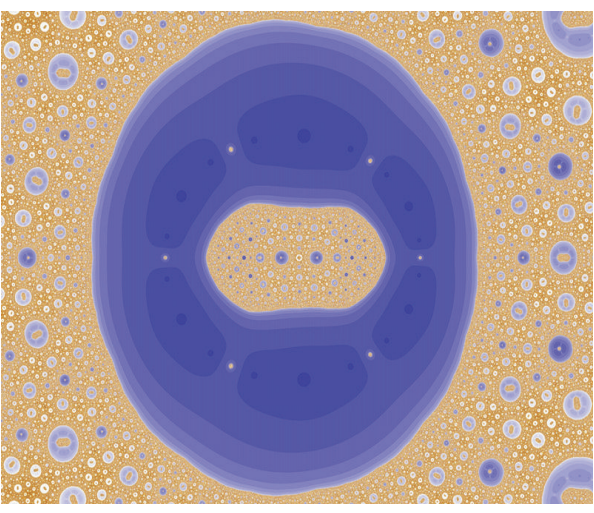

(a)

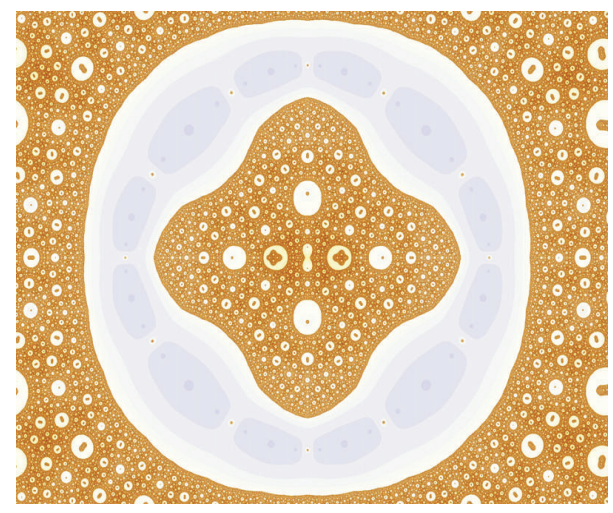

(b)

Figure 2: Two Fatou components of $\widetilde{R}(z, 0.004355)$ with connectivities 8 and 14 , respectively.

and for any $x \in[1,2]$, we have $|R(-x / t, t)|>3 / 2$; thus $\left|R\left(c_{0}, t\right)\right|>3 / 2$. By Lemma 5 , it follows that $c_{0} \notin D_{01}$. Moreover, three critical points $0,1 / t$ (twice) lie outside of $D_{01}$. Hence, $D_{01}$ contains at most four critical points. By Lemma 6, since the triply-connected domain $\Omega \subset D_{01}$ and $1 / t^{4} \in$ $J(R(z, t)) \cap\left\{\left|z-1 / t^{4}\right|<1 / 2 t^{4}\right\}$, then $\chi\left(D_{1}\right) \leq-1$. Applying the Riemann-Hurwitz formula to the threefold covering map $R: D_{1} \rightarrow D_{00}$, we have

$$
\chi\left(D_{1}\right)+\sum\left(k_{j}-1\right)=3 \chi\left(D_{00}\right)=3,
$$

so $\sum\left(k_{j}-1\right) \geq 4$. It follows that $\sum\left(k_{j}-1\right)=4$ and $\chi\left(D_{1}\right)=-1$, and thus $n\left(D_{01}\right)=3$.

By Lemma $5, \overline{D_{00}}$ is bounded. Since $\infty$ is a repelling fixed point, then each component in the preimage of $D_{00}$ is bounded. In fact, we have the following result.

Proposition 8. Each Fatou component of $R(z, t)$ is bounded.

Proof. By Proposition 7, $D_{01}$ contains four critical points and they tend to $z=0$ under $R^{n}(z, t)(n \rightarrow \infty)$. Note that $R(1 / t, t)=\infty$; then the dynamics of $R(z, t)$ are decided by the forward orbit of the critical point $c_{0}$ in Proposition 7 . If $\lim _{n \rightarrow \infty} R^{n}\left(c_{0}\right) \neq 0$, there exists at most one cycle of periodic components which is distinct from $D_{00}$ by Sullivan's theorem. Assume that this cycle exists, denoted by $U_{1}, \ldots, U_{p}$; then

$$
F(R(z, t))=\bigcup_{n=0}^{\infty} R^{-n}\left(D_{00}\right) \cup\left(\bigcup_{n=0}^{\infty} \bigcup_{i=1}^{p} R^{-n}\left(U_{i}\right)\right) .
$$

Otherwise, $F(R(z, t))=\cup_{n=0}^{\infty} R^{-n}\left(D_{00}\right)$.

Below we will prove that each component of $F(R(z, t))$ is bounded. From the above analysis, let $D$ be any component of $F(R(z, t))$; there exists $n \in \mathbb{N}$ such that $R^{n}(D)=D_{00}$ or $R^{n}(D)=U_{1}$ (if it exists). In order to show that $D$ is bounded, we need to prove that $\bar{U}_{1}$ is bounded. Suppose that $U_{1}, \ldots, U_{p}$ exist, and note that $\cup_{n=0}^{+\infty} R^{n}\left(c_{0}\right) \subset \mathbb{R} \cup\{\infty\}$; we have $U_{1}, \ldots, U_{p}$ which are (super)attracting or parabolic components by the Sullivan theorem, and thus $c_{0} \in \cup_{i=1}^{p} U_{i}$. Without loss of generality, $c_{0} \in U_{1}$. By Lemma $3, U_{i}(i=1, \ldots, p)$ is simply connected; then $\overline{U_{1}}$ is contained in the bounded component of $\mathbb{C}$.

Remark 9. By Proposition $8, z=\infty$ and its preimages are buried points; here a point $z_{0}$ in Julia set is called buried point if it is not on the boundary of any Fatou component.

By Lemma 6 and Propositions 7 and 8, let $M_{1}$ be the component of $\overline{\mathbb{C}} \backslash D_{01}$ which contains $1 / t^{4}$. Clearly, $M_{1} \neq\left\{1 / t^{4}\right\}$, and in the following, we denote $M=\operatorname{Int}\left(D_{01}\right) \backslash$ $\left(D_{01} \cup M_{1}\right)$; then $1 / t \in M$. Below we consider the connectivity of $R^{-1}\left(D_{01}\right)$.

Proposition 10. $R^{-1}\left(D_{01}\right)$ consists of three Fatou components with connectivities 5,3 , and 3 , respectively.

Proof. We claim that $R^{-1}\left(D_{01}\right)$ consists of three Fatou components. On one hand, since $R\left(\operatorname{Out}\left(D_{01}\right)\right)=R\left(M_{1}\right)=\operatorname{Out}\left(D_{00}\right)$ and $D_{01} \subset \operatorname{Out}\left(D_{00}\right)$, there exists at least one component of $R^{-1}\left(D_{01}\right)$ in Out $\left(D_{01}\right)$ and $M_{1}$, respectively. On the other hand, since $R(z, t)$ is monotone increasing from 0 to $+\infty$ for $z \in(0,1 / t)$ and $D_{01} \supset \Omega$ by Lemma 6 , there exists a unique component $D_{2}$ of $R^{-1}\left(D_{01}\right)$ with $D_{2} \cap(0,1 / t) \neq \emptyset$ and $D_{2} \subset M$. We claim that

$$
D_{2} \circlearrowleft \frac{1}{t}
$$

Assume that (15) is true (in what follows, we will return to the proof of this fact later in the proof), and by the definition of interior at the beginning of this section, $1 / t \in \operatorname{Int}\left(D_{2}\right)$. Since $1 / t$ is a critical point with multiplities 2 and $1 / t^{4}, \infty \notin$ $\operatorname{Int}\left(D_{2}\right)$, then $R(z, t)$ is a 3 -fold map from $\operatorname{Int}\left(D_{2}\right)$ to some neighborhood $U$ of $\infty$. Furthermore, we can easily deduce that $D_{2}$ is the unique component of $R^{-1}\left(D_{01}\right)$ in $\operatorname{Int}\left(D_{2}\right)$ (otherwise, $R(z, t): \operatorname{Int}\left(D_{2}\right) \rightarrow U$ is at least a 4-fold map; it is a contradiction). Hence, $\left.d\right|_{D_{2}}=3$ owing to $R(z, t): D_{2} \rightarrow$ $D_{01}$ which is a proper map. Obviously, we have $\left.d\right|_{R(z, t)}=5$, so the number of components of $R^{-1}\left(D_{01}\right)$ in $\operatorname{Out}\left(D_{01}\right)$ and $M_{1}$ is exactly one, respectively, denoted by $D_{1}$ and $D_{3}$. Clearly, $D_{1}, D_{2}$, and $D_{3}$ are mutually disjoint preimage components of $R^{-1}\left(D_{01}\right)$ and $\left.d\right|_{D_{1}}=\left.d\right|_{D_{3}}=1$. 
Below we prove (15). In fact, if $D_{2}$ does not surround $1 / t$, we distinguish two cases to discuss and get a contradiction.

(i) If $D_{2}$ does not surround $D_{00}$, it is easy to see that $R\left(\operatorname{Int}\left(D_{2}\right)\right)=\operatorname{Int}\left(D_{01}\right)$ and $D_{00} \subset \operatorname{Int}\left(D_{01}\right)$, and since $D_{2}$ has no pole, then $R^{-1}\left(D_{00}\right) \cap \operatorname{Int}\left(D_{2}\right) \neq \emptyset$, but it is a contradiction to Lemma 6.

(ii) If $D_{2} \circlearrowleft D_{00}$, note that $R(z, t)<0$ for $z \in\left(1 / t, 1 / t^{4}\right)$ and $\lim _{z \rightarrow 1 / t+} R(z, t)=-\infty$, and we can deduce that there exists at least one component (denoted by $\widehat{D_{2}}$ ) of $R^{-1}\left(D_{01}\right)$ with $\widehat{D_{2}} \cap\left(1 / t, 1 / t^{4}\right) \neq \emptyset$. Obviously, $\widehat{D_{2}} \subset$ $\operatorname{Out}\left(D_{01}\right)$ and $\widehat{D_{2}} \cap D_{2}=\emptyset$ since $D_{2}$ does not surround $1 / t$. If $\widehat{D_{2}} \circlearrowleft D_{00}$, then $\widehat{D_{2}} \circlearrowleft 1 / t$, and by a similar discussion as used in the case of $D_{2}$, it is easy to see that $\left.d\right|_{\widehat{D_{2}}}=3$, but it is a contradiction to the fact that both $M$ and $M_{1}$ contain some connected components of $R^{-1}\left(D_{01}\right)$. If $\widehat{D_{2}}$ does not surround $D_{00}$, we also get a contradiction by a similar discussion of case (i). Hence, we get $D_{2} \circlearrowleft 1 / t$.

Next we will acquire the connectivity of $D_{i}(i=1,2,3)$. Obviously, $c_{0} \notin D_{1} \cup D_{3}$, and by the Riemann-Hurwitz formula, $n\left(D_{1}\right)=n\left(D_{3}\right)=3$. Furthermore, we claim that the "free" critical point $c_{0}$ in Proposition 7 is not contained in $D_{2}$, and thus $n\left(D_{2}\right)=5$. In order to prove that $c_{0} \notin D_{2}$, we turn to show the stronger result as follows:

$$
\overline{D_{2}} \cap \mathbb{R}^{-}=\emptyset .
$$

Otherwise, assume that $x \in \overline{D_{2}} \cap \mathbb{R}^{-}$. Note that $t$ is a real parameter and $D_{2} \cap \mathbb{R}^{+} \neq \emptyset$; then $D_{2}$ is symmetric with respect to real axis $\mathbb{R}$. We choose a Jordan curve $\gamma$ in the interior of $D_{2}$ such that $\gamma$ is very close to $\partial \operatorname{Out}\left(D_{2}\right)$ and symmetric with respect to $\mathbb{R}$, and we take a point $z_{0}$ in $\gamma \cap\{z \mid z \in \mathbb{C}, \operatorname{Im} z \geq$ 0 \} such that $z_{0}$ is one of the nearest points from $x$; denote the arc of $\gamma$ from $\overline{z_{0}}$ to $z_{0}$ in counterclockwise direction by $\Gamma$. Moreover, we can also choose a Jordan $\operatorname{arc} \eta$ between $x$ and $z_{0}$ in $\gamma \cap\{z \mid z \in \mathbb{C}, \operatorname{Im} z \geq 0\}$ such that $\eta \cap \Gamma=\left\{z_{0}\right\}$. Set $\widetilde{\eta}=\{z \mid \bar{z} \in \eta\}$ and take $\widetilde{\Gamma}=\Gamma \cup \eta \cup \widetilde{\eta}$; then $\widetilde{\Gamma}$ is a Jordan curve in $\overline{D_{2}}$ and $\widetilde{\Gamma} \cap \partial D_{2} \subset\{x\}$. Since $N(R, \widetilde{\Gamma})=2, P(R, \widetilde{\Gamma})=3$, and $\arg R(z, t)$ changes by $-2 \pi$ as $z$ goes around $\widetilde{\Gamma}$ by argument principle, but $\arg R(z, t)$ changes by $-6 \pi$ since $\left.d\right|_{D_{2}}=3$; it is a contradiction. Hence, we get (16).

By a similar argument as the one used in (15), we can deduce that $D_{3} \circlearrowleft 1 / t^{4}$ and $D_{1} \circlearrowleft D_{01}$. In fact, it is decided by the "similarity" of the Julia sets $J(R(z, t))$. By the definition of $M_{1}$ and Lemma 6 , for any $x_{0} \in M_{1} \cap \mathbb{R}, x_{0}>1 / 2 t^{4}$. Let $x_{1} \in \mathbb{R}^{-}$be the largest point of $\partial D_{01}$ and let $x_{2} \in \mathbb{R}^{+}$ be the largest point of $\partial D_{00}$; by Lemma $5, R\left(x_{1}, t\right)=x_{2}<$ 2. Furthermore, by Lemma 6 and (16), the unique "free" critical point $c_{0}$ in Proposition 7 satisfies $c_{0} \in\left(x_{1}, 0\right)$. Note that $R(z, t)$ is monotone increasing in $\left(x_{1}, c_{0}\right)$ and monotone decreasing in $\left(c_{0}, 0\right)$; by a calculation, we can easily deduce that $\left|R\left(c_{0}, t\right)\right|<1 / t^{2}$ for any $x \in\left(-1 / t^{4}, 0\right)$. It is easy to see that $R(z, t)$ is monotone increasing in $(0,1 / t)$ from 0 to $+\infty$; then the equation $R(z, t)=x_{0}$ has only one real root in $\left(-1 / t^{4}, 1 / t^{4}\right)$ since $R(z, t)<0$ for $z \in\left(1 / t, 1 / t^{4}\right)$. Since

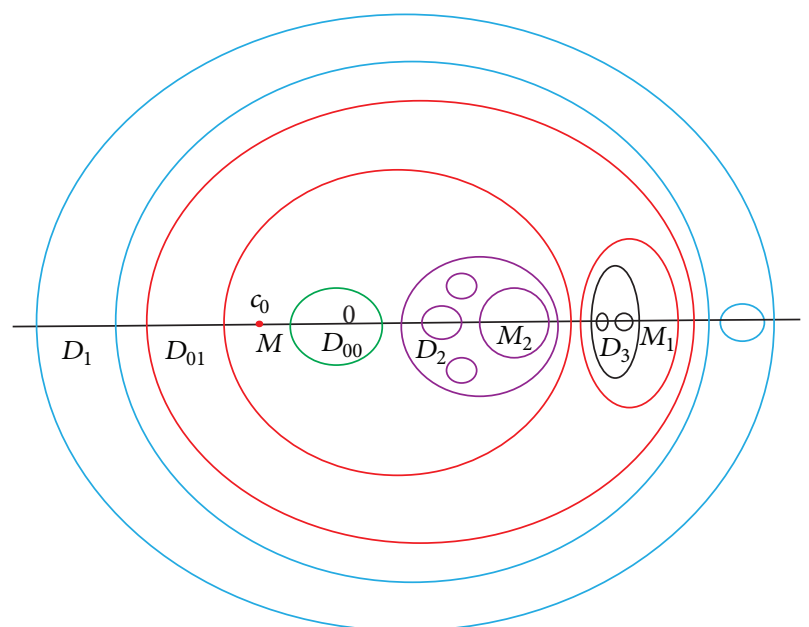

FIgURE 3: Schematic diagram of $\cup_{i=0}^{2} R^{-i}\left(D_{00}\right)$.

$D_{2} \subset M$ and $M \cap \mathbb{R} \subset\left(-1 / t^{4}, 1 / t^{4}\right)$, we can deduce that the number of bounded components of $\overline{\mathbb{C}} \backslash D_{2}$ which intersects with $\mathbb{R}$ is two (see Figure 3 ).

For any component $D$ in the preimage of $D_{i}(i=1,2,3)$, it is easy to see that $n(D) \geq 3$ by the Riemann-Hurwitz formula. Furthermore, the connectivity $n(D)$ is decided by the number of critical points in $D$ and local degree $\left.d\right|_{D}$. It is easy to see that there exists at most one Fatou component which contains the free critical point $c_{0}$; the following Proposition 13 shows that even if there is no critical point in $D$, its local degree may be larger than one. Therefore, we cannot give a complete description of connectivity of this family of rational maps. In order to get Proposition 13, we first consider the number of poles in $\operatorname{Int}(D)$.

Proposition 11. No preimage component of $D_{2}$ or $D_{3}$ surrounds $0,1 / t$ or $1 / t^{4}$.

Proof. We argue by contradiction and induction. Let $D$ be a preimage component of $D_{2}$ or $D_{3}$; we distinguish the following three cases to discuss.

(i) Suppose that $D \circlearrowleft 0$; it is easy to see that $D \circlearrowleft D_{00}$ since $0 \in D_{00}$ and $D \neq D_{00}$.

(ii) Suppose that $D \cup 1 / t$; then $D \subset M_{2}$ or $D \subset \operatorname{Out}\left(D_{2}\right)$. If $D \subset M_{2}$, since $R(z, t)>0$ for any $z \in D \cap(0,1 / t)(\neq \emptyset)$ and $R(z, t)<0$ for any $z \in D \cap\left(1 / t, 1 / t^{4}\right)(\neq \emptyset), R(D) \circlearrowleft D_{00}$ by symmetry. If $D \subset \operatorname{Out}\left(D_{2}\right)$, then $D \cup D_{00}$ or there exist two points $z_{1} \in(0,1 / t) \cap D$ and $z_{2} \in\left(1 / t, 1 / t^{4}\right) \cap D$. Since $R\left(z_{1}, t\right)>0$ and $R\left(z_{2}, t\right)<0$, then $R(D)$ surrounds $D_{00}$ by symmetry.

(iii) Suppose that $D \circlearrowleft 1 / t^{4}$; then either $D$ or $R(D)$ surrounds $D_{00}$ by the similar proof of (i) and (ii).

In all, if $D$ surrounds any of $0,1 / t$ or $1 / t^{4}$, then either $D$ or $R(D)$ surrounds $D_{00}$. Note that if $R(D)$ surrounds $D_{00}$, we have $D \varsubsetneqq R^{-1}\left(D_{2}\right)$ and $D \varsubsetneqq R^{-1}\left(D_{3}\right)$ since $D_{2} \cap \mathbb{R}^{-}=\emptyset$ and $D_{3} \subset M_{1}$. To get the conclusion in this proposition, it suffices to prove that no preimage component of $D_{2}$ or $D_{3}$ surrounds $D_{00}$. 
Below we prove that no preimage component of $D_{2}$ surrounds $D_{00}$ by induction.

Let $D$ be a component of $R^{-1}\left(D_{2}\right)$. Obviously, we have $D \cap$ $D_{00}=\emptyset$. Suppose that $D \cup D_{00}$; we will get contradictions by discussion.

(iv) Suppose that $D \subset \operatorname{Out}\left(D_{01}\right)$, and since $\left(-\infty,-1 / t^{4}\right) \cap$ $D \neq \emptyset$, we get $R(D) \cap \mathbb{R}^{-1} \neq \emptyset$ which contradicts with $D_{2} \cap$ $\mathbb{R}^{-1}=\emptyset$.

(v) Suppose that $D \subset M$, and choosing a Jordan curve $\gamma$ in $D$ such that $\gamma \circlearrowleft D_{00}$, it is easy to know that $N(R, \gamma)=2$ and $P(R, \gamma)=0$ or 3 . Since $R(D)=D_{2}$ and $D_{2}$ does not surround $D_{00}$, the contradiction can be deduced by argument principle.

Hence, any component $D$ of $R^{-1}\left(D_{2}\right)$ cannot surround $D_{00}$. Assume that any component of $R^{-n}\left(D_{2}\right)$ cannot surround $D_{00}$. Again, let $D$ be a component of $R^{-(n+1)}\left(D_{2}\right)$. Suppose that $D \cup D_{00}$; we still distinguish two cases to discuss.

(vi) Suppose that $D \subset \operatorname{Out}\left(D_{01}\right)$, and since $R(z, t)<0$ for any $z \in D \cap\left(-\infty,-1 / t^{4}\right)(\neq \emptyset)$ and $R(z, t)>0$ for any $z \in$ $D \cap\left(1 / t^{4},+\infty\right)(\neq \emptyset)$, then $R(D)$ surrounds $D_{00}$ by symmetry which contradicts with the assumption.

(vii) Suppose that $D \subset M$, and since $R(D)$ is a component of $R^{-n}\left(D_{2}\right)$ and $R(D) \neq D$, then $R(D)$ cannot surround $D_{00}$ by assumption. By a similar analysis as used in the case (v), we also deduce a contradiction.

Therefore, we get that no preimage component of $D_{2}$ surrounds $D_{00}$. By a similar discussion as the above used in $D_{2}$, any preimage component of $D_{3}$ cannot surround $D_{00}$.

However, the conclusion in Proposition 11 cannot fit for $D_{1}$. For simplicity, the symbol $(1 \times n)(n \in \mathbb{N})$ is $\underbrace{1 \cdots 1}_{n}$ and $D_{(1 \times 0) i}=D_{i}$ for $i=1,2,3$.

Proposition 12. Given that $n \in[1,+\infty)$, only one component of $R^{-n}\left(D_{1}\right)$ surrounds $0,1 / t$, and $1 / t^{4}$. Moreover, only one component of $R^{-n}\left(D_{1}\right)$ surrounds $1 / t$ but does not surround 0 and $1 / t^{4}$. In addition, only one component of $R^{-n}\left(D_{1}\right)$ surrounds $1 / t^{4}$ but does not surround 0 and $1 / t$.

Proof. By induction and a similar discussion as the one used in Proposition 10, it is easy to get the following conclusion. For any integer $n \in \mathbb{N}, R^{-1}\left(D_{(1 \times n)}\right)$ consists of three components: one is contained in $\operatorname{Out}\left(D_{(1 \times n)}\right)$, denoted by $D_{(1 \times(n+1))}$; one is contained in $M_{2}$, denoted by $D_{(1 \times n) 2}$; one is contained in $M_{1}$, denoted by $D_{(1 \times n) 3}$. Moreover, $D_{(1 \times(n+1))}$ ㄷ $D_{(1 \times n)}$ and $D_{(1 \times n) i}$ ฯ $D_{(1 \times(n+1)) i}(i=2,3)$. By a similar discussion as used in Proposition 11, we can deduce that any preimage component of $D_{(1 \times m) 2}$ or $D_{(1 \times m) 3}(m=1, \ldots, n-1)$ cannot surround 0 or $1 / t$. Since $R^{n}\left(D_{(1 \times n) i}\right)=D_{i}(i=1,2,3)$, then $D_{(1 \times(n+1))}, D_{(1 \times n) 2}$ and $D_{(1 \times n) 3}$ are satisfied with conditions of this proposition in turn as follows:

$$
D_{00} \longleftarrow D_{01} \longleftarrow\left\{\begin{array} { l } 
{ D _ { 2 } } \\
{ D _ { 1 } } \\
{ D _ { 3 } }
\end{array} \longleftarrow \left\{\begin{array} { l } 
{ D _ { 1 2 } } \\
{ D _ { 1 1 } } \\
{ D _ { 1 3 } }
\end{array} \longleftarrow \left\{\begin{array}{l}
D_{112} \\
D_{111} \\
D_{113}
\end{array} \cdots .\right.\right.\right.
$$

By Proposition 10, we can deduce that $n\left(D_{(1 \times n) 1}\right)=$ $n\left(D_{(1 \times n) 3}\right)=3, n\left(D_{(1 \times n) 2}\right)=5$ for any $n \in[1,+\infty)$. However, for the other components in the preimage of $D_{i}(i=1,2,3)$, we have the following result.

Proposition 13. Given that $n \in \mathbb{N}$, let $D$ be a component of preimage of $D_{(1 \times n) 2}$ or $D_{(1 \times n) 3}$, and if $c_{0} \in \operatorname{Out}(D)$, then $\left.d\right|_{D}=$ 1 ord $\left.\right|_{D}=2$.

Proof. By Propositions 11 and 12, $\operatorname{Int}(D)$ contains no pole of $R(z, t)$, and thus $R(\operatorname{Int}(D))=\operatorname{Int} R(D)$ and $R$ is a proper map in $\operatorname{Int}(D)$. We distinguish three cases to discuss the local degree of $D$ as follows.

(1) If $c_{0} \in \operatorname{Out}(D)$, then $\left.d\right|_{\operatorname{Int}(D)}=1$ by the RiemannHurwitz formula. Obviously, $\left.d\right|_{D}=1$.

(2) If $c_{0} \in D$, then $\left.d\right|_{D} \geq 2$. By the Riemann-Hurwitz formula, $\left.d\right|_{\operatorname{Int}(D)}=2$, so $\left.d\right|_{D}=2$.

(3) If $c_{0} \in \operatorname{Int}(D) \backslash D$, then $\left.d\right|_{\operatorname{Int}(D)}=2$ by the RiemannHurwitz formula. Furthermore, we can deduce that $\left.d\right|_{D}=2$. Otherwise, $\left.d\right|_{D}=1$. Note that $\operatorname{Int}(D)$ has no poles of $R(z, t)$, and for any bounded components of $\overline{\mathbb{C}} \backslash R(D)$, the forward components of it in $\operatorname{Int}(D)$ are corresponding to all bounded components of $\overline{\mathbb{C}} \backslash D$. Hence, $R^{-1}(R(D)) \cap \operatorname{Int}(D)=D$; it is a contradiction to $\left.d\right|_{\operatorname{Int}(D)}=2$.

Proof of Theorem 1. (1) It is an immediate result of Propositions 7 and 10.

(2) We show that $R(z, 1 / 20)$ has a Fatou component with connectivity 9.

Put $t_{0}=1 / 20$, and by Proposition 7 , the "free" critical point $c_{0}$ satisfies $c_{0} \in\left(-2 / t_{0}+5 t_{0}^{2},-2 / t_{0}+6 t_{0}^{2}\right)$. Set $R(x)=R\left(x, t_{0}\right), r_{1}(x)=x^{2}, r_{2}(x)=1+t_{0}^{12} x^{3}, r_{3}(x)=$ $\left(1-t_{0} x\right)^{3}, r_{4}(x)=1-t_{0}^{4} x(x \in \mathbb{R})$; then $R(x)=$ $\left(r_{1}(x) r_{2}(x)\right) /\left(r_{3}(x) r_{4}(x)\right)$. Put $I_{1}=\left[-2 / t_{0}+5 t_{0}^{2},-2 / t_{0}+6 t_{0}^{2}\right]$, and for any $x_{1} \in I_{1}$, we have

$$
\begin{aligned}
& \frac{r_{1}\left(-2 / t_{0}+6 t_{0}^{2}\right) r_{2}\left(-2 / t_{0}+5 t_{0}^{2}\right)}{r_{3}\left(-2 / t_{0}+5 t_{0}^{2}\right) r_{4}\left(-2 / t_{0}+5 t_{0}^{2}\right)} \\
& \quad \leq R\left(x_{1}\right) \leq \frac{r_{1}\left(-2 / t_{0}+5 t_{0}^{2}\right) r_{2}\left(-2 / t_{0}+6 t_{0}^{2}\right)}{r_{3}\left(-2 / t_{0}+6 t_{0}^{2}\right) r_{4}\left(-2 / t_{0}+6 t_{0}^{2}\right)} .
\end{aligned}
$$

Furthermore, we can deduce that

$$
R\left(x_{1}\right) \in[59.237,59.252]:=I_{2}, \quad \forall x_{1} \in I_{1} .
$$

If necessary, we enlarge or reduce the length of corresponding interval, by a similar discussion as the one used in inequality 5, as follows:

$$
\begin{aligned}
& R\left(x_{2}\right) \in[-465.125,-464.357]:=I_{3}, \quad \forall x_{2} \in I_{2} ; \\
& R\left(x_{3}\right) \in[15.065,15.188]:=I_{4}, \quad \forall x_{3} \in I_{3} ; \\
& R\left(x_{4}\right) \in[15108,16564]:=I_{5}, \quad \forall x_{4} \in I_{4} ;
\end{aligned}
$$




$$
\begin{aligned}
& R\left(x_{5}\right) \in[-0.714,-0.445]:=I_{6}, \quad \forall x_{5} \in I_{5} ; \\
& R\left(x_{6}\right) \in[0.178,0.478]:=I_{7}, \quad \forall x_{6} \in I_{6} .
\end{aligned}
$$

For example,

$$
\frac{r_{1}(59.252) r_{2}(59.252)}{r_{3}(59.237) r_{4}(59.252)} \leq R\left(x_{2}\right) \leq \frac{r_{1}(59.237) r_{2}(59.237)}{r_{3}(59.252) r_{4}(59.237)} \text {. }
$$

By Lemmas 5 and $6, I_{7} \cup I_{6} \subset D_{00}$ and $I_{5} \subset D_{01}$. Note that $I_{4}=[15.065,15.188]$; then $I_{4} \subset D_{2}$. Let $D$ be the Fatou component that contains $c_{0}$, and since $R\left(c_{0}\right)>0$, then $R(D) \cap \mathbb{R}^{-}=\emptyset$ by Proposition 11, and thus $c_{0} \in \operatorname{Out}(R(D))$. As $R\left(z, t_{0}\right)=-1 /\left(t_{0}\right)^{4}$ has an approximate root 22.9777(>1/t $\left.t_{0}\right)$, we have $R(D) \subset \operatorname{Out}\left(D_{2}\right)$. By Proposition 12, we can deduce that $R^{2}(D)$ cannot surround $D$. By Proposition $13,\left.d\right|_{D}=2$ and $d_{R^{n}(D)}=1(n=1,2)$, and thus $n(R(D))=n\left(R^{2}(D)\right)=5$, $n(D)=9$ by the Riemann-Hurwitz formula.

\section{Conflict of Interests}

The authors declare that there is no conflict of interests regarding the publication of this paper.

\section{Acknowledgments}

The authors would like to thank the referees for their valuable suggestions for improving this paper. This research was supported by the National Natural Science Foundation of China (nos. 11371363, 11231009, and 11261002), the Fundamental Research Funds for the Central Universities (no. 2009QS15), and the construct program of the key discipline in Hunan province.

\section{References}

[1] D. Sullivan, "Quasiconformal homeomorphisms and dynamics. I. Solution of the Fatou-Julia problem on wandering domains," Annals of Mathematics, vol. 122, no. 3, pp. 401-418, 1985.

[2] I. N. Baker, J. Kotus, and Y. N. Lü, "Iterates of meromorphic functions. III. Preperiodic domains," Ergodic Theory and Dynamical Systems, vol. 11, no. 4, pp. 603-618, 1991.

[3] A. F. Beardon, Iteration of Rational Functions, vol. 132 of Graduate Texts in Mathematics, Springer, Berlin, Germany, 1991.

[4] J. Y. Qiao and J. Y. Gao, "The connectivity numbers of Fatou components of rational mappings," Acta Mathematica Sinica, vol. 47, no. 4, pp. 625-628, 2004 (Chinese).

[5] M. Stiemer, "Rational maps with Fatou components of arbitrary connectivity number," Computational Methods and Function Theory, vol. 7, no. 2, pp. 415-427, 2007.

[6] L. Carleson and T. W. Gamelin, Complex Dynamics, Tracts in Mathematics, Springer, Berlin, Germany, 1991.

[7] J. Milnor, Dynamics in One Complex Variable, vol. 160 of Annals of Mathematics Studies, Princeton University Press, Princeton, NJ, USA, 3rd edition, 2006.

[8] F. Przytycki, "Iterations of rational functions: which hyperbolic components contain polynomials?" Fundamenta Mathematicae, vol. 149, no. 2, pp. 95-118, 1996. 


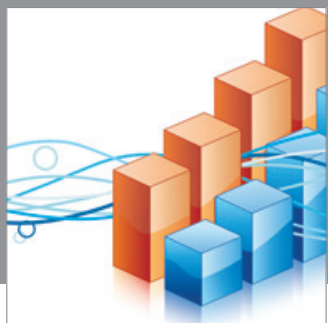

Advances in

Operations Research

mansans

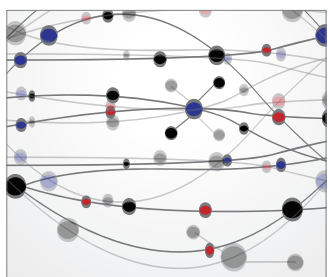

The Scientific World Journal
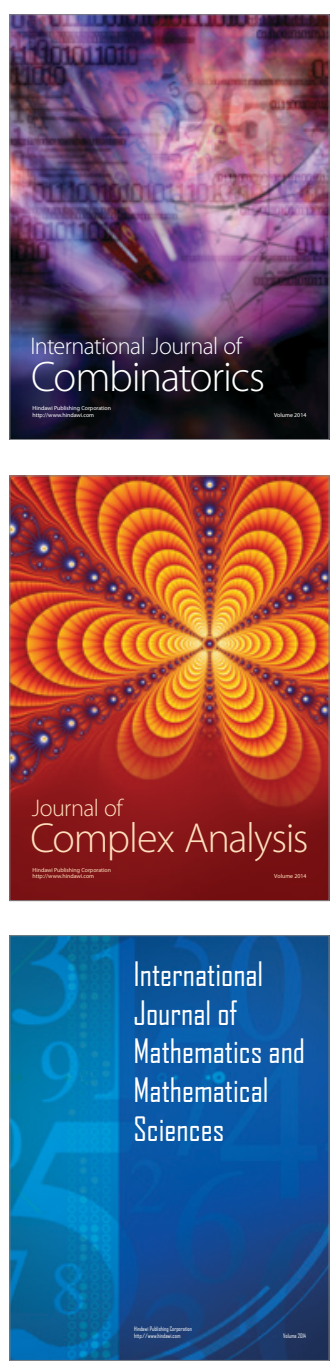
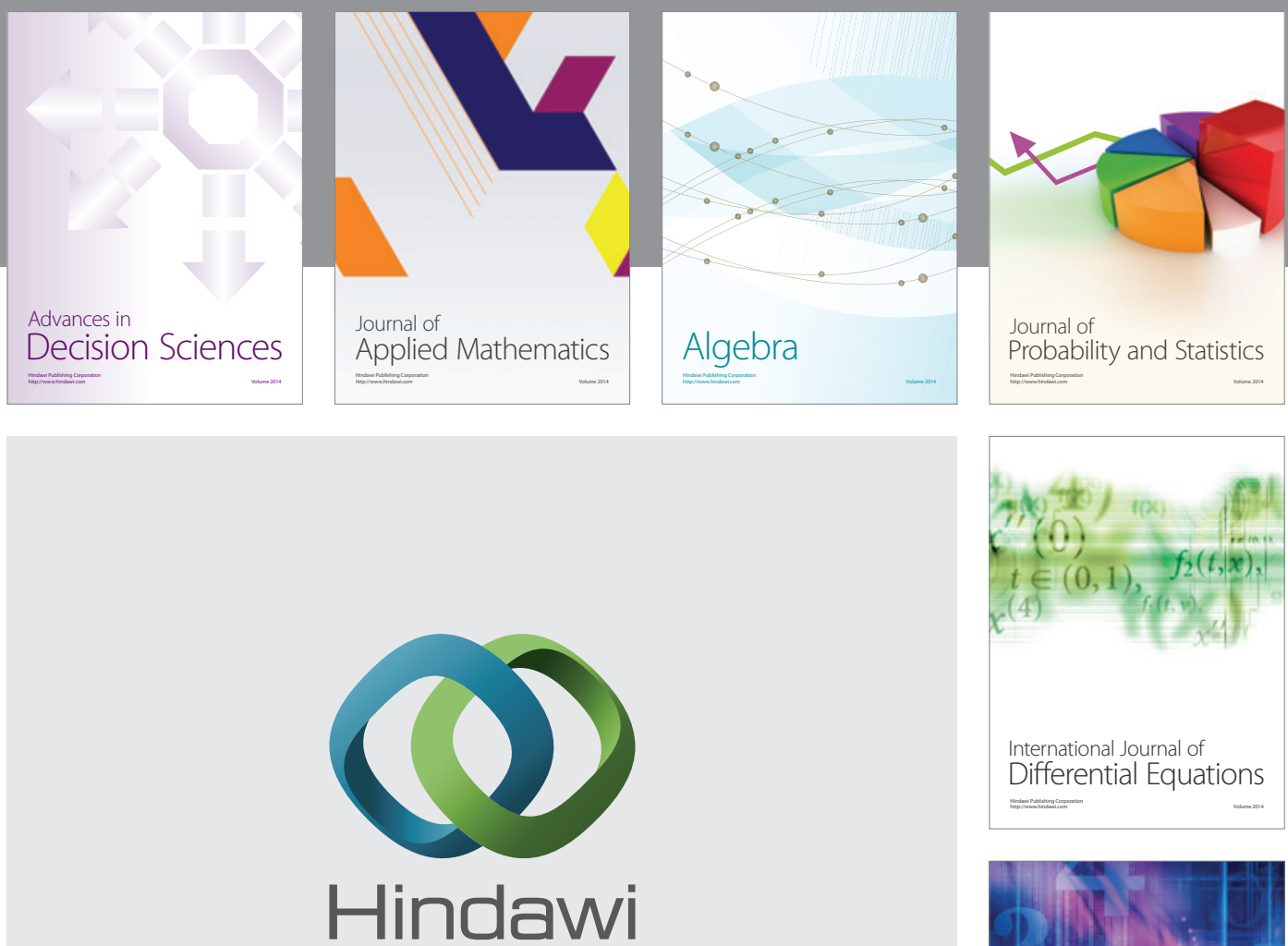

Submit your manuscripts at http://www.hindawi.com
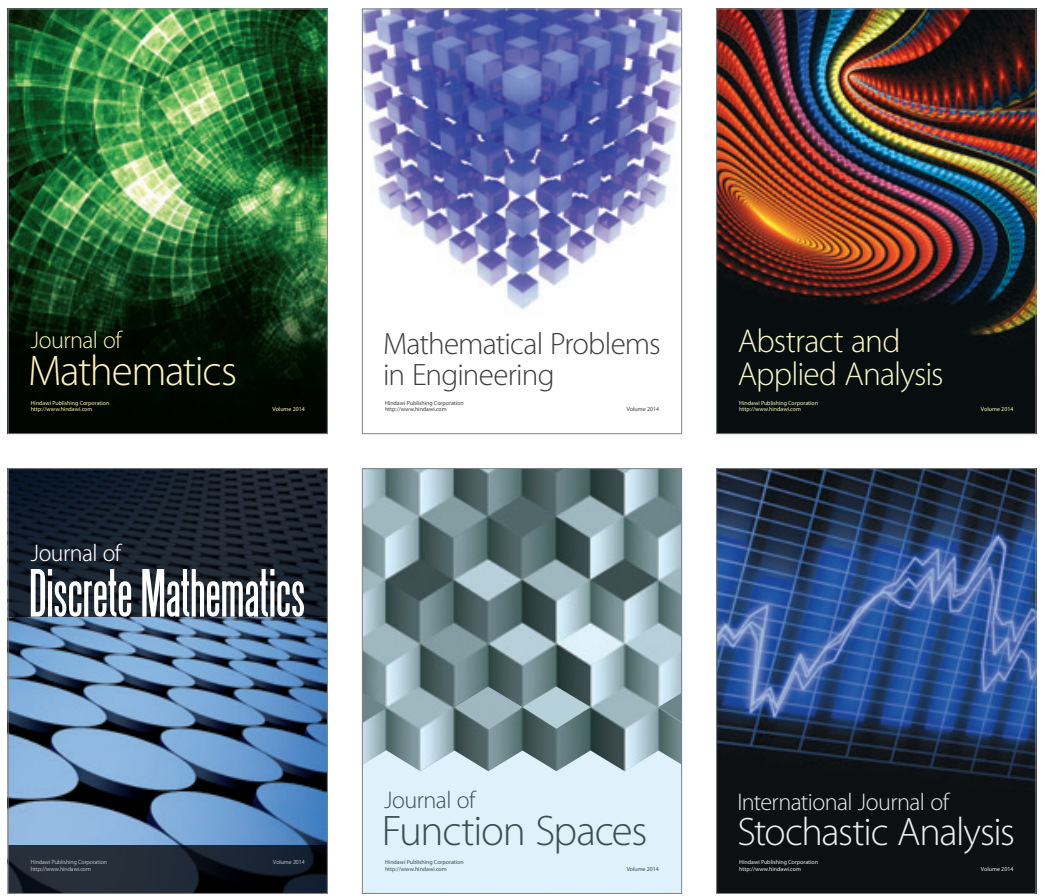

Journal of

Function Spaces

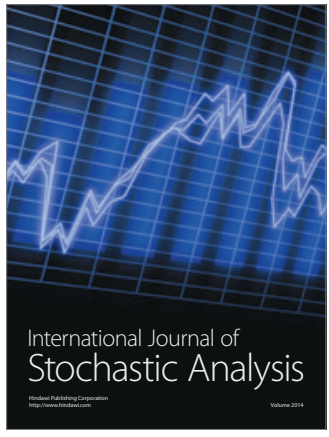

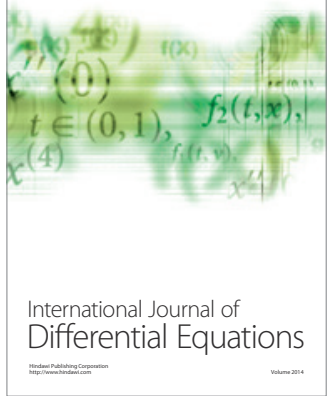
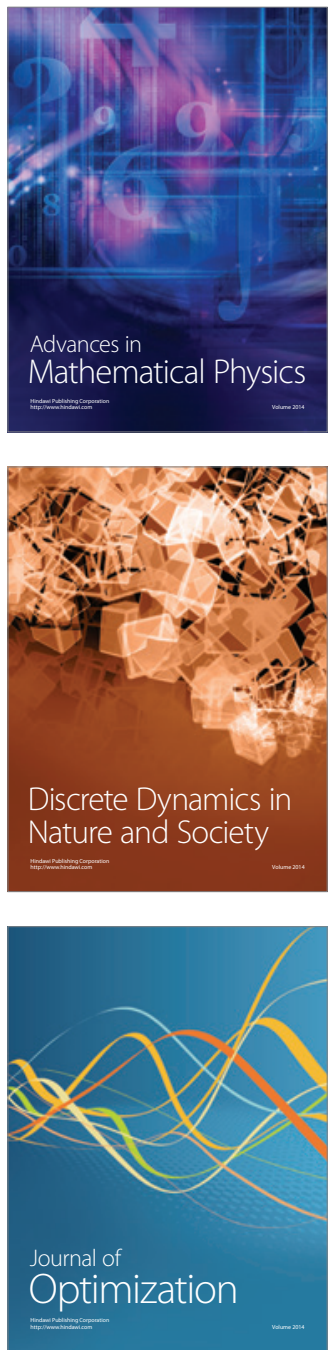